\title{
HOMOTOPY TORSION IN CODIMENSION TWO KNOTS ${ }^{1}$
}

\section{W. SUMNERS}

I. Introduction. The homology theory of the infinite cyclic covering space of a codimension two knot complement is reasonably well known, but less is known concerning the homotopy theory of the knot complement itself. If $k: S^{n} \rightarrow S^{n+2}$ is a smooth embedding, let $S=S^{n+2}-k\left(S^{n}\right)$ denote the open knot complement. One of the module structures on the homotopy of $S$ is the natural structure over the ring $\Gamma=$ integral group ring of $\pi_{1}(S)$. We say that $\pi_{i}(S)(i \geqq 2)$ has $\Gamma$ torsion if there exists $0 \neq c \in \pi_{i}(S), 0 \neq w \in \Gamma$ such that $0=w \cdot c \in \pi_{i}(S)$.

This paper gives a technique for constructing slice (null-cobordant) knots with $\Gamma$-torsion. The homotopy torsion in these examples is related to the homology torsion in $\tilde{S}$, the infinite cyclic cover of $S$. If $\Lambda$ denotes the integral group ring of the infinite cyclic group (generated by $t)$, then $H_{i}(\tilde{S} ; Z)$ is a finitely-generated $\Lambda$-module for all $i$. In the construction, the $\Lambda$-generator of $H_{i}(\tilde{S} ; Z) \neq 0$ is an embedded $S^{i}$. Furthermore the $\Lambda$-torsion in $H_{i}(\tilde{S} ; Z)$ arises from an embedded bounded punctured $D^{i+1}$. This punctured $D^{i+1}$ has as boundary a finite disjoint union of copies of $S^{i}$, and piping them together inside $D^{i+1}$ yields an embedded sphere which bounds an embedded disc. This gives a nontrivial $\Gamma$-relation in $\pi_{i}(S)$.

If $h_{1}: \pi_{1}(S) \rightarrow H_{1}(S ; Z)$ is the Hurewicz homomorphism, then $h_{1}$ extends uniquely to the ring homomorphism $\bar{h}_{1}: \Gamma \rightarrow \Lambda$. $\Lambda$ is thought of as the ring of Laurent polynomials in a variable $t$ with integer coefficients. If $0 \neq \lambda \in \Lambda$, then $\Lambda / \lambda$ denotes the cokernel of the injection

$$
\begin{aligned}
& \stackrel{\lambda}{\rightarrow} \Lambda \\
& 1 \mapsto \lambda
\end{aligned}
$$

(see Levine [5]).

We prove the following

THEOREM 1. Given any polynomial $F(t) \in \Lambda$ such that $F(1)= \pm 1$, then there exists a smooth slice knot $\left(S^{4}, k S^{2}\right)$ such that

(i) $H_{1}(\widetilde{S} ; Z) \cong_{\Lambda} \Lambda / F(t), H_{2}(\tilde{S} ; Z) \cong{ }_{\Delta} \Lambda / F\left(t^{-1}\right)$;

Presented to the Society, January 24, 1968; received by the editors January 27, 1969, and, in revised form, August 8, 1969.

1 This research supported by NSF Grant GP-5458. 
(ii) $\pi_{2}(S)$ has $\Gamma$-torsion $w \cdot c=0$ where $0 \neq c \in \pi_{2}(S)$ and $0 \neq w \in \Gamma$;

(iii) $\bar{h}_{1}(w)=F\left(t^{-1}\right)$, and $h_{2}: \pi_{2}(\tilde{S}) \rightarrow H_{2}(\tilde{S} ; Z)$ the Hurewicz homomorphism then $h_{2}(c)$ is the $\Lambda$-generator of $H_{2}(\tilde{S} ; Z)$.

One of the knots produced in Theorem 1 is a counterexample to a theorem announced by C. H. Giffen.

The techniques developed in the proof of Theorem 1 can be used to prove the following

Theorem 2. Given any polynomial $F(t) \in \Lambda, F(1)= \pm 1$, and integers $n$, $p$ such that $n \geqq 2,1 \leqq p<(n+1) / 2$ then there exists a smooth slice knot $\left(S^{n+2}, k S^{n}\right)$ such that

(i) $H_{p}(\widetilde{S} ; Z) \cong_{\Lambda} \Lambda / F(t), H_{n+1-p}(\tilde{S} ; Z) \cong{ }_{\Lambda} \Lambda / F\left(t^{-1}\right), H_{r}(\widetilde{S} ; Z)=0$ $r \neq 0, p, n+1-p$

(ii) $\pi_{n+1-p}(S)$ has $\Gamma$-torsion $w \cdot c=0,0 \neq c \in \pi_{n+1-p}(S), 0 \neq w \in \Gamma$;

(iii) $\bar{h}_{1}(w)=F\left(t^{-1}\right)$, and $h_{n+1-p}: \pi_{n+1-p}(\tilde{S}) \rightarrow H_{n+1-p}(\tilde{S} ; Z)$ the Hurewicz homomorphism then $h_{n+1-p}(c)$ is the $\Lambda$-generator of $H_{n+1-p}(\tilde{S} ; Z)$;

(iv) if $p \geqq 2$ then

$$
\begin{gathered}
\Gamma \stackrel{\bar{h}_{1}}{\cong} \Lambda, \\
\pi_{i}(S) \cong \pi_{i}\left(S^{1}\right), \quad i \leqq p-1,
\end{gathered}
$$

and

$$
\pi_{p}(S) \underset{\Lambda}{\cong} \pi_{p}(\tilde{S}) \underset{\Lambda}{\stackrel{h_{p}}{\rightleftarrows}} H_{p}(\tilde{S} ; Z) .
$$

Note that Theorem 2 avoids the case $n=2 q+1(q \geqq 2)$ and $p=q$. In this case it turns out that

$$
\begin{aligned}
& \pi_{i}(S) \cong \pi_{i}\left(S^{1}\right), \quad i \leqq q-1, \\
& \pi_{q}(S) \cong \pi_{q}(\tilde{S}) \underset{\Lambda}{\stackrel{h_{q}}{\cong}} H_{q}(\tilde{S} ; Z) \underset{\Lambda}{\cong} \Lambda / F(t) F\left(t^{-1}\right) .
\end{aligned}
$$

This arises because the construction relies on a certain duality between dimensions $p$ and $n+1-p$, and in the above mentioned case, dimension $q$ is selfdual.

We can prove a similar result for ball pairs. Let $k^{\prime}: B^{n+2} \rightarrow B^{n+3}$ denote proper smooth embedding of balls, and $B=B^{n+3}-k^{\prime}\left(B^{n+1}\right)$ denote the open knot complement, and $\widetilde{B}$ the infinite cyclic cover of $B$. 
Theorem 3. Given $F(t) \in \Lambda$, and integers $n$, $p$ such that $2 \leqq n, 2 \leqq p \leqq n$ then there exists a smooth knotted ball pair $\left(B^{n+3}, k^{\prime} B^{n+1}\right)$ such that

(i) $H_{p}(\widetilde{B} ; Z) \cong{ }_{\Lambda} \Lambda / F(t), H_{r}(\widetilde{B} ; Z)=0, \quad r \neq 0, p$;

(ii) $\pi_{p}(B) \cong_{\Lambda} \pi_{p}(\tilde{B}) \underset{\Im_{\Lambda}}{\stackrel{h_{p}}{\longrightarrow}} H_{p}(\tilde{B} ; Z), \pi_{i}(B) \cong \pi_{i}\left(S^{1}\right), i \leqq p-1$.

II. The construction. We will begin with a special case of the proof of Theorem 1. Suppose that $F(t)=(2-t)$. The knot is constructed in the same manner as those in [3], [7], [8], [9]. Let $\left(B^{5}, B^{3}\right)$ denote the unknotted ball pair, and $B^{*}=B^{5}-B^{3}$ the open complement. Let $f: S^{0} \rightarrow \partial B^{*}$ be an embedding, and attach a 1 -handle to $B^{*}$ by $f$, rounding corners to obtain the smooth manifold $K=B^{*} \cup_{f} h^{1}$ [Figure 1].

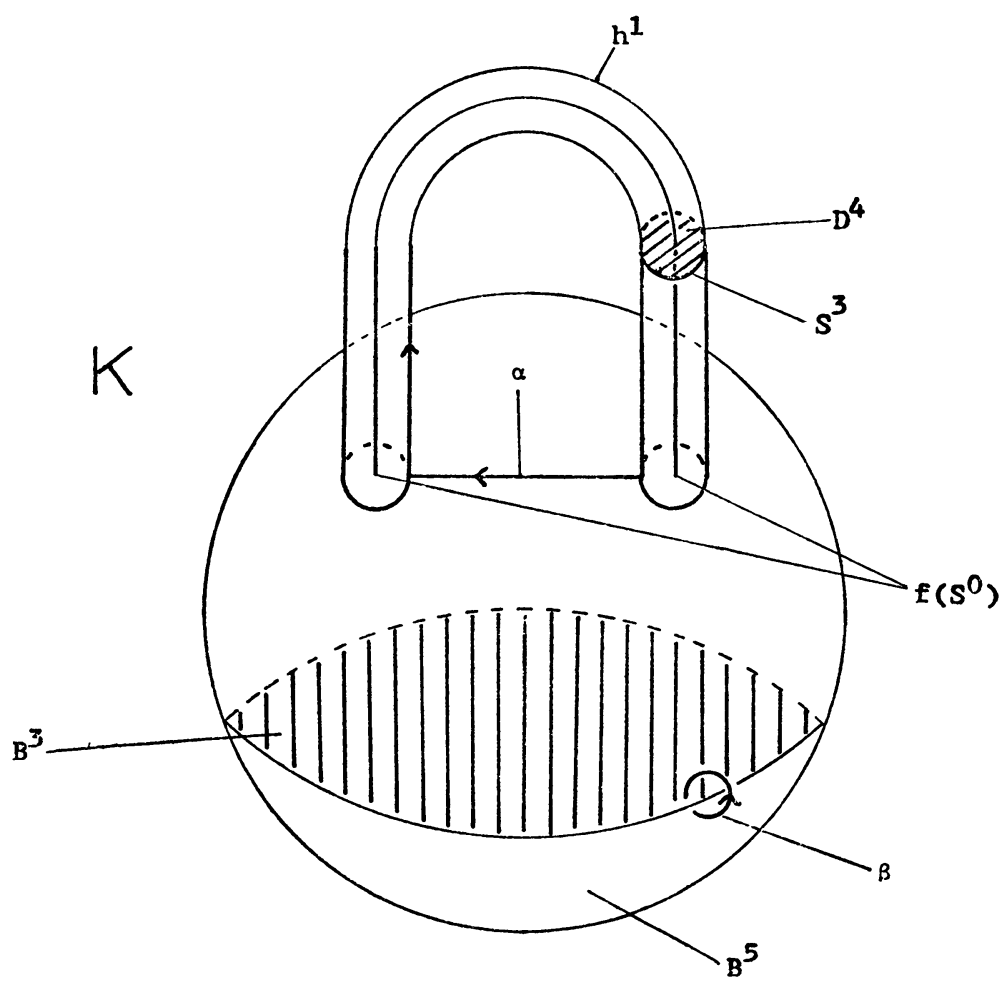

Figure 1

Let $\approx$ denote diffeomorphism, $\simeq$ denote homotopy equivalence, and $\checkmark$ denote wedge product. Then 


$$
\begin{aligned}
& K \approx S^{1} \times D^{4}-B^{3} \quad K \simeq S^{1} \bigvee S^{3}, \\
& \partial K \approx S^{1} \times S^{3}-S^{2} \quad \partial K \simeq S^{1} \vee S^{1} \vee S^{3} .
\end{aligned}
$$

As in Figure 1, let $\alpha$ denote the generator of $\pi_{1}(\partial K)$ which goes around the handle and $\beta$ the generator which links once the "flat" $S^{2}$ in $\partial K$. Let $g: S^{1} \rightarrow \partial K$ be the smooth embedding given in Figure 2. $g\left(S^{1}\right)$ is an embedding in the homotopy class of $\alpha^{2} \beta \alpha^{-1} \beta^{-1} \in \pi_{1}(\partial K)$. If $*$ is

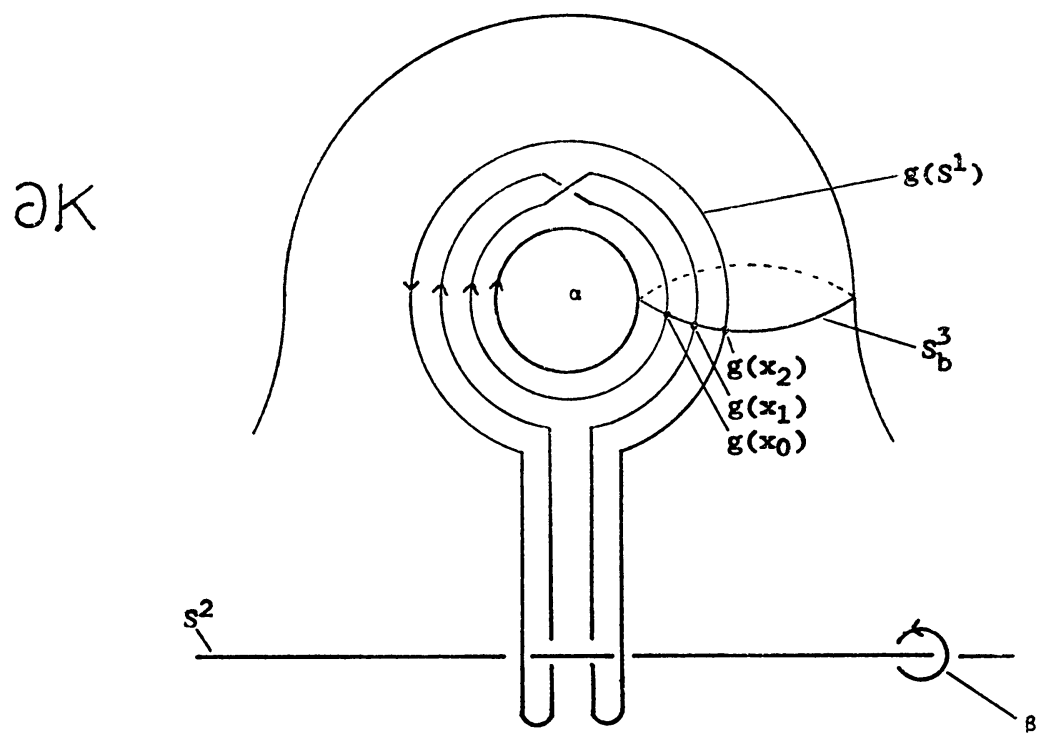

FIGURE 2

the base point of $S^{3}$, then in the product structure of $\partial K \approx S^{1} \times S^{3}-S^{2}$, $S^{1} \times *$ is an embedding in the homotopy class of $\alpha$, and we will henceforth identify $\alpha$ with $S^{1} \times *$. Now from Figure 2, $g\left(S^{1}\right)$ is clearly diffeotopic to $\alpha$ in $\partial K \cup S^{2}$. Since $\alpha$ has a trivial normal bundle in $\partial K$ then so does $g\left(S^{1}\right)$. Add a 2-handle to $K$ by $g$, rounding corners to obtain $B=K \cup_{0} h^{2}=B * \cup_{f} h^{1} \cup_{\jmath} h^{2}$. Now $B \cup B^{3}=B^{*} \cup B^{3} \cup_{f} h^{1}$ $\cup_{0} h^{2} \approx B^{5} \cup_{f} h^{1} \cup_{\alpha} h^{2} \approx B^{5}$. The last diffeomorphism is from the handle cancellation theorem, since the attaching sphere of the 2-handle intersects the belt sphere of the 1-handle transversely at one point. Therefore $B$ is a knot complement; that is $B=B^{5}-k^{\prime} B^{3}$ for some smooth proper embedding $k^{\prime}: B^{3} \rightarrow B^{5}$. Furthermore $\partial B=S$ $=S^{4}-k S^{2}$ is the complement of a smooth slice knot, where $k=k^{\prime} \mid \partial B^{3}$.

III. Homotopy calculations. We have $B \simeq S^{1} \bigvee S^{1} \cup D^{2}$, and $\pi_{1}(B)$ 
$=\left(a, \beta \mid \alpha^{2} \beta \alpha^{-1} \beta^{-1}\right)$. By considering the trace of the surgery associated with the addition of the 2-handle to $K$, we see that the inclusion $i: S \rightarrow B$ induces the isomorphism

$$
i_{*}: \pi_{1}(S) \stackrel{\cong}{\rightarrow} \pi_{1}(B) .
$$

That is, let $W$ be the trace (Figure 3 ), such that $\partial_{-} W=\partial K$ and $\partial_{+} W=S$. Now if $D^{2}$ represents the core of $h^{2}$ and $D^{3}$ represents the transverse disc of $h^{2}$, then $\partial K \cup D^{2} \simeq W \simeq S \cup D^{3}$. So $\pi_{1}(S) \cong \pi_{1}\left(\partial K \cup D^{2}\right)$. By considering the trace of the surgery associated with the addition of the 1-handle to $B^{*}$, we can see that $\pi_{1}\left(\partial K \cup D^{2}\right) \cong \pi_{1}\left(K \cup D^{2}\right)$ $=\pi_{1}(B)$.

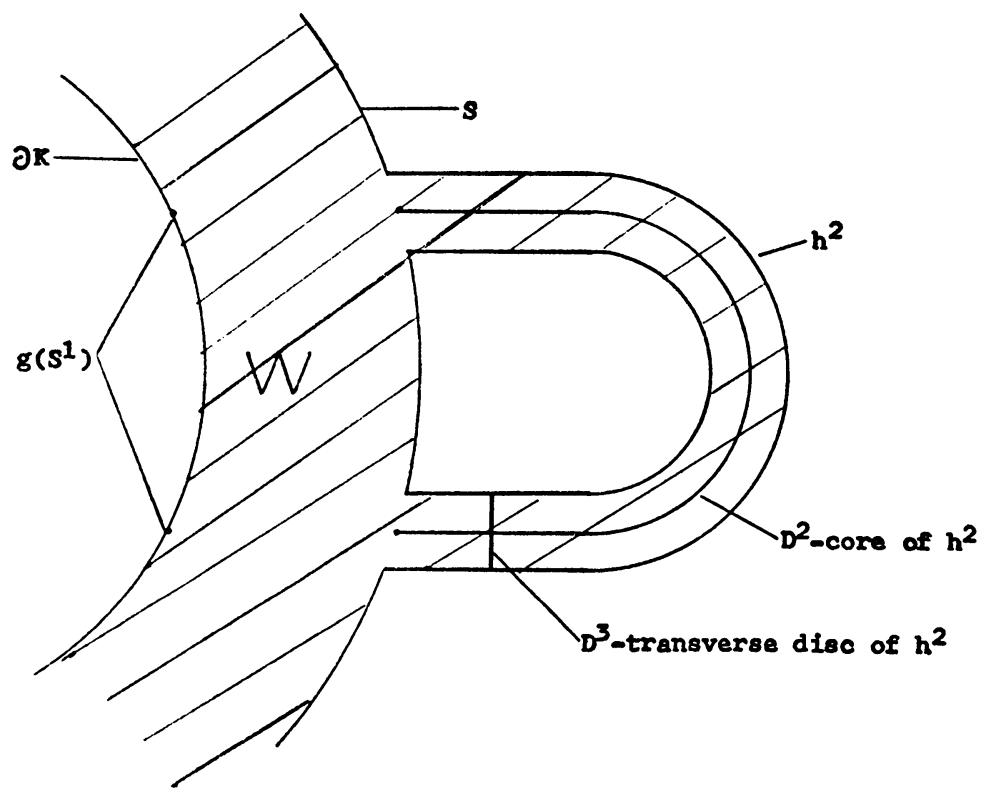

Figure 3

We will now consider the structure of $\pi_{2}(S)$ as a module over $\Gamma$. Let $b$ be the base point of $S^{1}$. In the product structure of $\partial K$ let $S_{b}^{3}=b \times S^{3}$ be the belt sphere of $h^{1}$. [See Figure 2.] Suppose that $x_{0}, x_{1}, x_{2} \in S^{1}$ are 3 distinct points such that $S_{b}^{3} \cap g\left(S^{1}\right)=g\left(x_{0}\right) \cup g\left(x_{1}\right) \cup g\left(x_{2}\right)$. Let $G: S^{1} \times D^{3} \rightarrow \partial K$ be a trivialization of the tubular neighborhood of $g\left(S^{1}\right)$ in $\partial K$ such that $G\left(S^{1} \times D^{3}\right) \cap S_{b}^{3}=G\left(x_{0} \times D^{3}\right) \cup G\left(x_{1} \times D^{3}\right) \cup G\left(x_{2} \times D^{3}\right)$. Now $\partial\left(S_{b}^{3} \cap S\right)=d_{0} \cup d_{1} \cup d_{2}$ where $d_{i}=\partial G\left(x_{i} \times D^{3}\right), i=0,1,2$ are em- 
bedded 2-spheres. That is, $\left(S_{b}^{3} \cap S\right)$ is a punctured $D^{3}$. Figure 4 shows the situation in $\partial K$ near $S_{b}^{3}$. The hatched area is $S_{b}^{3} \cap S$. With choice of paths to the base point $*$ as given in Figure $4, d_{i} \in \pi_{2}(S)$. Furthermore, $d_{1}=\alpha^{-1} \cdot d_{0}$ where $\cdot$ denotes $\pi_{1}(S)$-action on $\pi_{2}(S)$. This can be

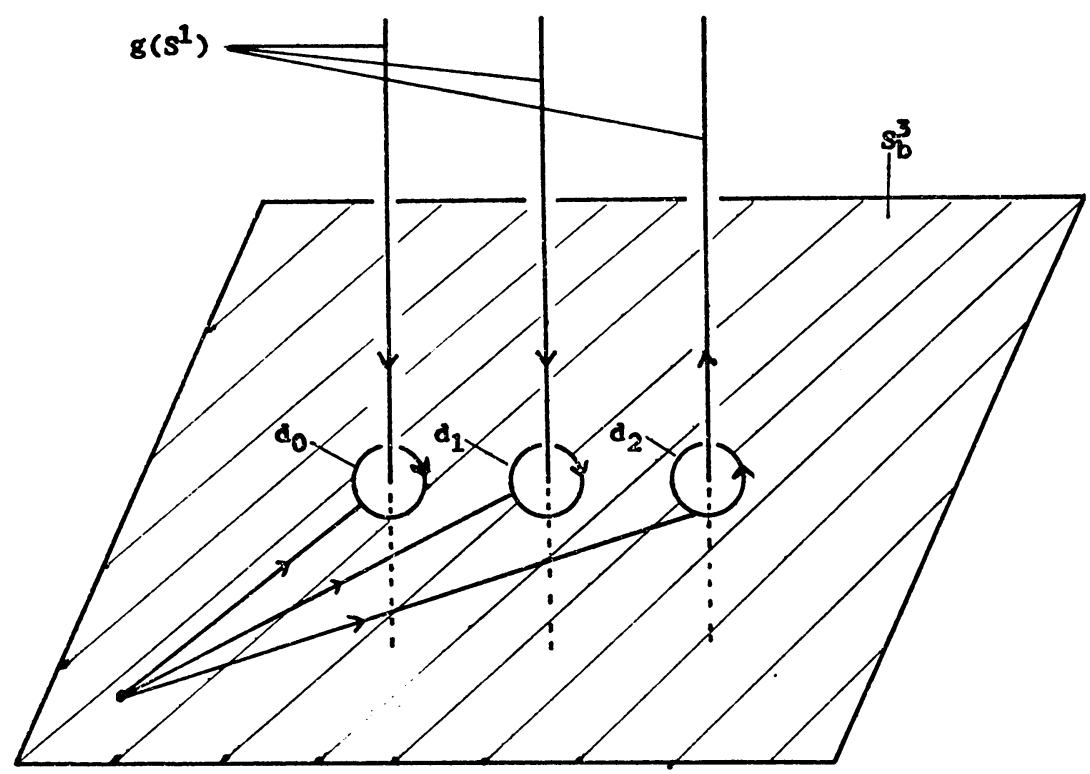

FIGURE 4

seen by moving $d_{0}$ around the boundary of the tubular neighborhood of $g\left(S^{1}\right)$ until it coincides (except for the path to $*$ ) with $d_{1}$, and then comparing the resulting paths to the base point under $\pi_{1}(S)$-action. Similarly, $d_{2}=\beta^{-1} \cdot d_{1}=\beta^{-1} \cdot\left(\alpha^{-1} \cdot d_{0}\right)$. Now changing the orientation of $d_{2}$, we can pipe the boundary components of $S_{b}^{3} \cap S$ together inside $S_{b}^{3} \cap S$ (Figure 5), obtaining an embedding in the homotopy class of $d_{0}+d_{1}-d_{2}=\left(1+\alpha^{-1}-\beta^{-1} \alpha^{-1}\right) \cdot d_{0} \in \pi_{2}(S)$. Clearly this sphere bounds an embedded $D^{3}$, so $\left(1+\alpha^{-1}-\beta^{-1} \alpha^{-1}\right) \cdot d_{0}=0 \in \pi_{2}(S)$. As will be shown later, $d_{0} \neq 0$ in $\pi_{2}(S)$ because $\tilde{d}_{0}$, a lift of $d_{0}$ in the infinite cyclic cover $\tilde{S}$, generates $H_{2}(\tilde{S} ; Z) \neq 0$ as a $\Lambda$-module. Furthermore $\bar{h}_{1}\left(1+\alpha^{-1}-\beta^{-1} \alpha^{-1}\right)=2-t^{-1} \neq 0$ in $\Lambda$, so $\left(1+\alpha^{-1}-\beta^{-1} \alpha^{-1}\right) \neq 0$ in $\Gamma$. Hence $\pi_{2}(S)$ has $\Gamma$-torsion.

More generally, if $F(t) \in \Lambda$ and $F(t)=\sum_{i=0}^{m} a_{i} t^{i}\left(a_{0}>0\right)$ then the construction proceeds exactly as before, with the exception that the 2-handle is attached by the element 


$$
\alpha^{a_{0}} \beta \alpha^{a_{1}} \beta \alpha^{a_{2}} \beta \cdots \beta \alpha^{a_{m} \beta^{-m}} \in \pi_{1}(\partial K) .
$$

(This is the formula produced in [7].) In this case the element $w \in \Gamma$ producing the torsion is

$$
\begin{aligned}
w= & \sum_{k=0}^{a_{0}-1} \alpha^{-k}+\operatorname{sgn} a_{1}\left[\left(\sum_{k=0}^{a_{1}-1} \alpha^{-k}\right) \beta^{-1} \alpha^{a_{0}-1}\right]+\cdots \\
& +\operatorname{sgn} a_{m}\left[\left(\sum_{k=0}^{\left|a_{m}\right|-1} \alpha^{-k}\right) \beta^{-1} \alpha^{\left|a_{m}-1\right|-1} \beta^{-1} \cdots \beta^{-1} \alpha^{a_{0}-1}\right]
\end{aligned}
$$

with the convention that if $a_{q}=0$ then $\operatorname{sgn} a_{q}=0$ and $\alpha^{\left|a_{q}\right|-1}=1$.

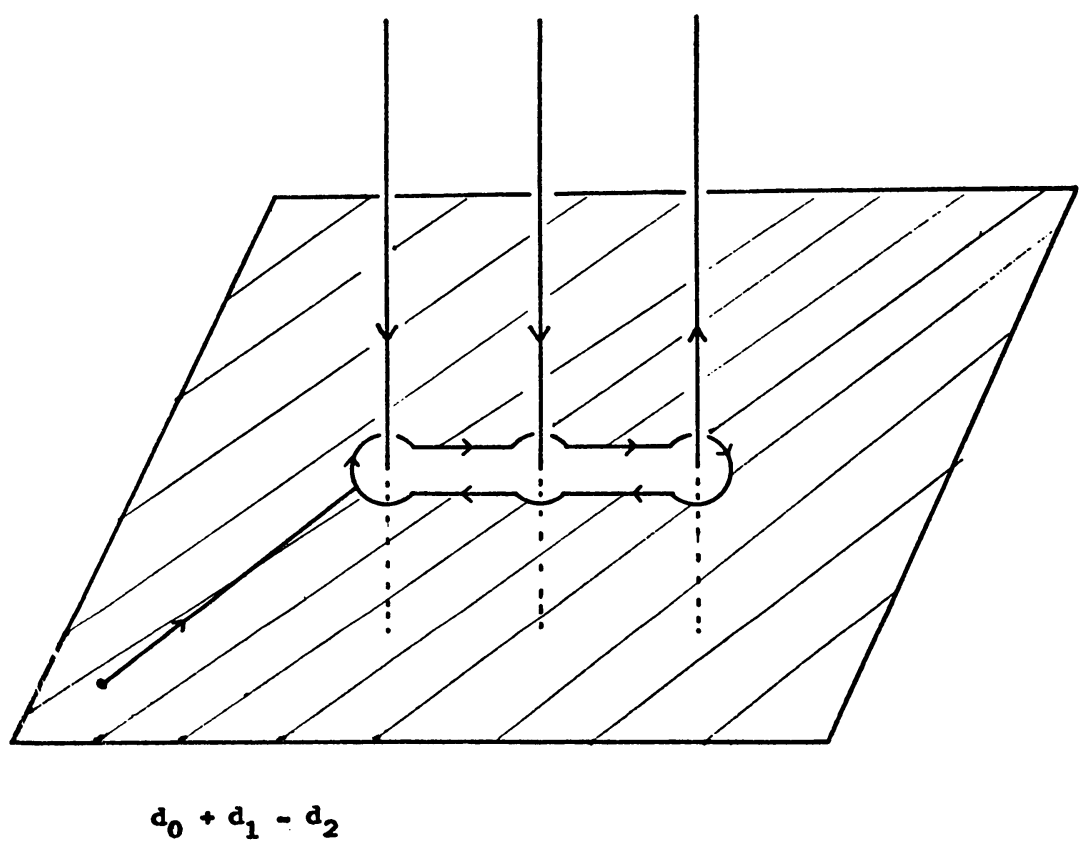

Figure 5

IV. The counterexample. Giffen [2, pp. 191] has made the following statement (restated for the smooth case):

"Let $S=S^{4}-k\left(S^{2}\right)$ be a smooth knot complement. Then $\pi_{2}(S)$ is the free Abelian group generated by the symbols $a_{c}$, where $1 \neq c$ $\in\left[\pi_{1}(S), \pi_{1}(S)\right]$ the commutator subgroup of $\pi_{1}(S)$. The action of $\pi_{1}(S)$ on $\pi_{2}(S)$ is that induced by basis permutation 


$$
P_{h}: a_{c} \rightarrow a_{h c h}^{-1}
$$

for $h \in \pi_{1}(S) . "$

Consider the knot produced in detail in the special case of Theorem 1. If the above statement is correct, then $d_{0} \in \pi_{2}(S)$ has an expansion as a finite linear combination of nontrivial elements of $\left[\pi_{1}(S), \pi_{1}(S)\right]$ (dropping $a_{c}$ and keeping $c$ ), that is $d_{0}=\sum_{i=1}^{q} m_{i} \xi_{i}$ where $\left\{m_{i}\right\}$ are integers and $\xi_{i} \in\left[\pi_{1}(S), \pi_{1}(S)\right]$. Further $\xi_{i} \neq \xi_{j}$ for $i \neq j$, and $m_{i} \neq 0$ for all $i$. It turns out in this case that $\left[\pi_{1}(S), \pi_{1}(S)\right]$ is Abelian and $\alpha^{-1} \in\left[\pi_{1}(S), \pi_{1}(S)\right]$. This will be proved in the next section. Allowing this, we compute the torsion relation in terms of the basis and the action: $\alpha^{-1} \in\left[\pi_{1}(S), \pi_{1}(S)\right]$ and action by conjugation means that the action of $\alpha^{-1}$ on $\pi_{2}(S)$ is trivial, so

$$
\left(1+\alpha^{-1}-\beta^{-1} \alpha^{-1}\right) \cdot d_{0}=\left(2-\beta^{-1}\right) \cdot d_{0}=0 .
$$

Or

$$
2 \sum_{i=1}^{q} m_{i} \xi_{i}-\sum_{i=1}^{q} m_{i} \beta^{-1} \xi_{i} \beta=0 .
$$

Since the action of $\pi_{1}(S)$ is by permutation on the basis elements, (1) can hold iff the action of $\beta^{-1}$ on $\left\{\xi_{i}\right\}_{i=1}^{q}$ is permutation within the set. So we can take it instead to be a permutation $\rho$ on the indexing set of the coefficients-that is if $\beta^{-1} \xi_{i} \beta=\xi_{j}$ then $m_{i} \beta^{-1} \xi_{i} \beta$ becomes $m_{\rho(j)} \xi_{j}$. So (1) is true iff the coefficients in each coordinate are zero; or $2 m_{i}-m_{\rho(i)}=0$ for all $i$. But, as a permutation on $q$ objects, $\rho$ has finite order, say $r \geqq 1$. Then $m_{1}=(1 / 2) m_{\rho(1)}=(1 / 4) m_{\rho^{2}(1)}=\cdots$ $=(1 / 2)^{r} m_{\rho^{r}(1)}=(1 / 2)^{r} m_{1}$. Since $m_{1} \neq 0$ then $1=(1 / 2)^{r}$, a contradiction.

What seems to be wrong with Giffen's statement is the description of the action of $\pi_{1}(S)$ on $\pi_{2}(S)$. For a correct description of the structure of $\pi_{2}(S)$ as a $\Gamma$-module in the case of fibered knots, see [1].

V. The infinite cyclic cover $\tilde{S}$. In order to complete the proof of Theorem 1, we will construct the infinite cyclic covering space $\tilde{S}$ of $S$, and study its homotopy and homology. We will produce a handlebody decomposition for $\widetilde{B}$ (the infinite cyclic cover of $B$ ), and the induced covering of $S$ will be $\tilde{S}$. The unknotted open complement $B^{*}=B^{5}-B^{3}$ fibers over $S^{1}$ with fiber $\hat{D}^{4}$, a half-closed $D^{4}$. That is, $\hat{D}^{4}$ has a closed $D^{3}$ (corresponding to the unknotted $B^{3}$ ) removed from its boundary. So the infinite cyclic cover of $B^{*}$ is $\widetilde{B}^{*}=R^{1} \times \hat{D}^{4}$. The attaching sphere $f\left(S^{0}\right)$ of $h^{1}$ lifts to a family of embedded spheres $f_{i}: S^{0} \rightarrow \partial \widetilde{B}^{*}$, where $i$ is associated with $t^{i} \in J(t)$ - the infinite cyclic group of covering transformations of $\widetilde{B}^{*}$. Add the family of 1 -handles $\left\{h_{i}^{1}\right\}$ to $\widetilde{B}^{*}$ by $f_{i}$ to get $\tilde{K}$, an infinite cyclic cover of $K$. Repeat the 
process for the 2 -handles to get $\widetilde{B}$. Figure 6 shows $\partial \widetilde{K}$ with the family $\left\{g_{i}\right\}$ of attaching spheres for the $\left\{h_{i}^{2}\right\}$. Let $\widetilde{S}_{0}=\partial \tilde{K}$, and $\widetilde{S}=\partial \widetilde{B}$ be the induced infinite cyclic cover of $S$. All integral homology groups

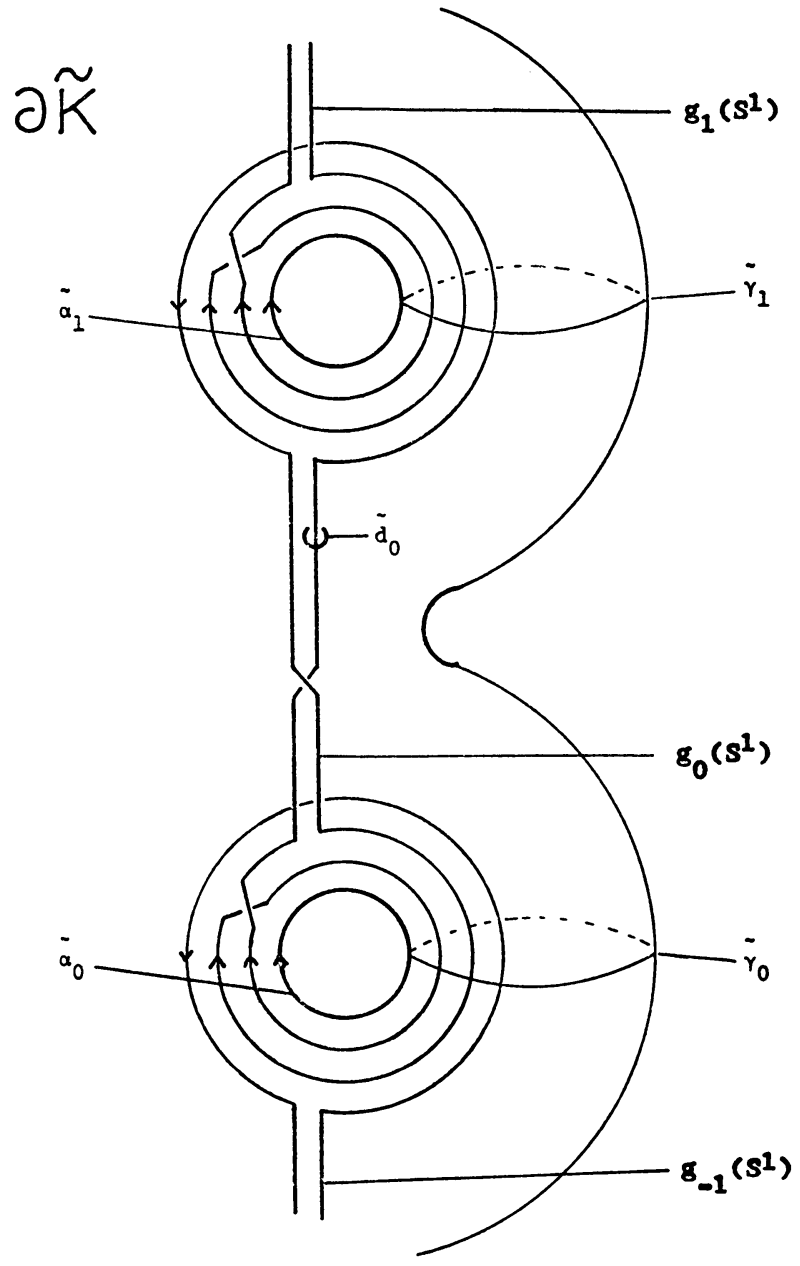

FigURE 6

in this situation are finitely-generated $\Lambda$-modules, and the exact sequences which follow are taken to be exact sequences of $\Lambda$-modules. The calculations follow those in [5], [8].

$$
\begin{aligned}
H_{i}\left(\tilde{S}_{0}\right) & =\Lambda & & i=1,3, \\
& =0 & & \text { otherwise. }
\end{aligned}
$$


Let $G_{i}: S^{1} \times D^{3} \rightarrow \widetilde{S}_{0}$ be a trivialization of the tubular neighborhood of the attaching sphere $g_{i}\left(S^{1}\right)$ of $h_{i}^{2}$, where $G_{i}$ is a lift of $G: S^{1} \times D^{3} \rightarrow \partial K$. Let $\widetilde{S}_{1}=\widetilde{S}_{0}-U_{i} G_{i}\left(S^{1} \times D^{3}\right)$ where upper bar denotes topological closure. By excision

$$
\begin{aligned}
H_{i}\left(\widetilde{S}_{0}, \widetilde{S}_{1}\right) & =\Lambda & & i=3,4 \\
& =0 & & \text { otherwise. }
\end{aligned}
$$

Likewise

$$
\begin{aligned}
H_{i}\left(\tilde{S}, \widetilde{S}_{1}\right) & =\Lambda & & i=2,4 \\
& =0 & & \text { otherwise. }
\end{aligned}
$$

Let $\Lambda(\tilde{\alpha})$ denote the free $\Lambda$-module generated by the symbol $\tilde{\alpha}$. Let $\tilde{\alpha}_{0}$ denote the lift of $\alpha$ at level $t^{0}$ in $\tilde{S}$ [Figure 6]. Let $\tilde{\gamma}_{0}$ be the lift of $S_{b}^{3}$ at level $t^{0}$ in $\tilde{S}$. Let $c_{0}=G\left(x_{0} \times D^{3}\right), \tilde{c}_{0}=G_{0}\left(x_{0} \times D^{3}\right)$ and $\tilde{d}_{0}=\partial \tilde{c}_{0}$ $=G_{0}\left(x_{0} \times S^{2}\right)$, the lift of $d_{0}$. With this choice of generators we have $H_{1}\left(\tilde{S}_{0}\right)=\Lambda\left(\tilde{\alpha}_{0}\right), H_{3}\left(\tilde{S}_{0}\right)=\Lambda\left(\tilde{\gamma}_{0}\right), H_{3}\left(\tilde{S}_{0}, \tilde{S}_{1}\right)=\Lambda\left(\tilde{c}_{0}\right), H_{2}\left(\tilde{S}, \tilde{S}_{1}\right)=\Lambda\left(\tilde{e}_{0}\right)$ where $\tilde{e}_{0}$ is the lift at level $t^{0}$ of the image of the core of $h^{2}$ after it has been pushed out onto the boundary of the handle.

Consider the exact sequence for the pair $\left(\widetilde{S}_{0}, \widetilde{S}_{1}\right)$ :

$$
\begin{aligned}
& H_{3}\left(\widetilde{S}_{0}\right) \stackrel{\cdot\left\{g_{i}\right\}}{\longrightarrow} H_{3}\left(\widetilde{S}_{0}, \widetilde{S}_{2}\right) \stackrel{\partial}{\rightarrow} H_{2}\left(\tilde{S}_{1}\right) \rightarrow 0 \\
& \| \\
& \Lambda\left(\tilde{\gamma}_{0}\right) \\
& \Lambda\left(\tilde{c}_{0}\right)
\end{aligned}
$$

where the homomorphism $\cdot\left\{g_{i}\right\}$ is as follows:

$$
\tilde{\gamma}_{0} \stackrel{\cdot\left\{g_{i}\right\}}{\longrightarrow}\left[\sum_{t^{i} \in J(t)} t^{1}\left\langle\tilde{\gamma}_{0} \cdot g_{i}\right\rangle\right] \tilde{c}_{0}
$$

where $\left\langle\tilde{\gamma}_{0} \cdot g_{i}\right\rangle$ is the intersection number of $\tilde{\gamma}_{0}$ and $g_{i}\left(S^{1}\right)$. (See [4, p. 515].) In this case $\cdot\left\{g_{i}\right\}\left(\tilde{\gamma}_{0}\right)=F\left(t^{-1}\right) \tilde{c}_{0}$. So $\cdot\left\{g_{i}\right\}$ is a monomorphism, and $H_{2}\left(\widetilde{S}_{1}\right)$ is presented as a $\Lambda$-module by the $1 \times 1$ matrix $F\left(t^{-1}\right)$. Moreover, the $\Lambda$-generator of $H_{2}\left(\tilde{S}_{1}\right)$ is $\partial \tilde{c}_{0}=\tilde{d}_{0}$.

Consider now the exact sequence for the pair $\left(\widetilde{S}, \widetilde{S}_{1}\right)$ :

$$
0 \rightarrow H_{2}\left(\tilde{S}_{1}\right) \rightarrow H_{2}(\tilde{S}) \rightarrow H_{2}\left(\tilde{S}, \tilde{S}_{1}\right) \stackrel{\partial}{\rightarrow} H_{1}\left(\tilde{S}_{1}\right)
$$


Since $\partial \tilde{e}_{0}=F(t) \tilde{\alpha}_{0}$, then $\partial$ is a monomorphism and $H_{2}\left(\tilde{S}_{1}\right) \cong H_{2}(\tilde{S})$. Therefore $\tilde{d}_{0} \neq 0$ in $H_{2}(\tilde{S})$; and hence $d_{0} \neq 0$ in $\pi_{2}(S)$. This completes the proof of Theorem 1 .

Since $\tilde{S}$ is the infinite cyclic cover of $S$, then $\pi_{1}(\tilde{S}) \cong\left[\pi_{1}(S), \pi_{1}(S)\right]$. From Figure 6 for $F(t)=(2-t)$, we see that $\pi_{1}(\tilde{S})$ is isomorphic to the group on infinitely many generators $\left\{\tilde{\alpha}_{i}\right\}$, with infinitely many relations $\left\{\tilde{\alpha}_{i}^{2} \tilde{\alpha}_{i+1}^{-1}=1\right\}$, which is Abelian because the generators commute. Furthermore, with correct choice of base point in $\tilde{S}, \tilde{\alpha}_{0}$ is the lift of $\alpha \in \pi_{1}(S)$, hence $\alpha^{-1} \in\left[\pi_{1}(S), \pi_{1}(S)\right]$. This completes the counterexample.

Theorem 2 is proved by construction in much the same way as Theorem 1, and is in fact easier to do when $p>1$. If $p=1$, the construction is exactly the same as in Theorem 1, only it takes place on the unknotted ball pair $\left(B^{n+3}, B^{n+1}\right)$. If $p>1$, then attach $h^{p}$ to $B^{*}=B^{n+3}-B^{n+1}$ by a nullisotopic $S^{p-1}$ in $\partial B^{*}$, obtaining $K=B^{*} \cup h^{p}$. As before

$$
\begin{array}{rlrl}
K & \approx S^{p} \times D^{n+3-p}-B^{n+1} & K & \simeq S^{1} \bigvee S^{p} \\
\partial K & \approx S^{p} \times S^{n+2-p}-S^{n} & \partial K & \simeq S^{1} \bigvee S^{p} \bigvee S^{n+2-p} .
\end{array}
$$

Attach $h^{p+1}$ to $K$ by an embedding in the homotopy class of $F(t) \cdot \alpha$, where $\alpha$ generates $\pi_{p}(\partial K)$. The knot produced has all the characteristics listed in Theorem 2.

Theorem 3 is proved in exactly the same way as Theorem 2 . The sphere pairs constructed in Theorem 2 bound ball pairs. These ball pairs have the characteristics listed in Theorem 3. However, the hypothesis of Theorem 3 allows $(n+1) / 2 \leqq p \leqq n$. For $p$ in this range (in and above the middle dimension of the boundary sphere pair), the construction can still be done. (See [8].)

Theorem 3 provides a generalization of the results of T. M. Price [6]. Using Zeeman's twist-spinning, he produces a $\left(B^{6}, k^{\prime} B^{4}\right)$ with boundary $\left(S^{5}, k S^{3}\right)$ such that $\pi_{1}\left(B^{6}-k^{\prime}\left(B^{4}\right)\right)=Z$ but $\pi_{1}\left(S^{5}-k\left(S^{3}\right)\right)$ $\neq Z$. Theorem 3 improves this example by one dimension, i.e. $\left(S^{4}, k S^{2}\right)$ $=\partial\left(B^{5}, k^{\prime} B^{3}\right)$ such that $\pi_{1}\left(B^{5}-k^{\prime}\left(B^{3}\right)\right)=Z, \pi_{1}\left(S^{4}-k\left(S^{2}\right)\right) \neq Z$. Furthermore, $\pi_{1}\left(S^{4}-k\left(S^{2}\right)\right)$ and $\pi_{2}\left(B^{5}-k^{\prime}\left(B^{3}\right)\right)$ can be specified as in Theorems 2 and 3 .

\section{REFERENCES}

1. J. J. Andrews and D. W. Sumners, On higher-dimensional fibered knots, Florida State Univ., Tallahassee, Florida (preprint).

2. C. H. Giffen, "On aspherical embeddings of 2 -spheres in the 4-sphere," in Topology seminar, Wisconsin, 1965, Princeton Univ. Press, Princeton, N. J., 1966, pp. 189-195. MR 34 \#1974. 
3. J. F. P. Hudson and D. W. Sumners, Knotted ball pairs in unknotted sphere pairs, J. London Math. Soc. 41 (1966), 717-722. MR 36 \#876.

4. M. A. Kervaire and J. W. Milnor, Groups of homotopy spheres. I, Ann. of Math. (2) 77 (1963), 504-537. MR 26 \#5584.

5. J. Levine, Polynomial invariants of knots of codimension two, Ann. of Math. (2) 84 (1966), 537-554. MR 34 \#808.

6. T. M. Price, A knotted cell pair with knot group $Z$, Illinois J. Math. 12 (1968), 201-204. MR 37 \#2219.

7. D. W. Sumners, Higher-dimensional slice knots, Bull. Amer. Math. Soc. 72 (1966), 894-897. MR 35 \#3690.

8. - Higher-dimensional slice knots, Ph.D. Thesis, Cambridge University, Cambridge, 1967.

9. C. T. C. Wall, On some knotted spheres and a theorem of B. Mazur, Cambridge University, Cambridge, 1964 (mimeographed).

Florida State University 